\title{
DNA DETECTION USING PROGRAMMED BILAYER NANOPORES
}

Ramkumar B ${ }^{1}$, Sohini Pal ${ }^{1}$, Himanshu Joshi ${ }^{2}$, Banani Chakraborty ${ }^{3}$, Akshay Naik ${ }^{1}$, Manoj Varma ${ }^{1}$, Prabal K. Maiti ${ }^{2}$

${ }^{1}$ Centre for Nano Science and Engineering, Indian Institute of Science, Bangalore 560012

${ }^{2}$ Department of Physics, Indian Institute of Science, Bangalore 560012

${ }^{3}$ Department of Chemical Engineering, Indian Institute of Science, Bangalore 560012

\begin{abstract}
:
Pore-functionalization has been explored by several groups as a strategy to control DNA translocation through solid-state nanopores. Here we present a hybrid nanopore system consisting of single-layer graphene and a DNA origami layer to achieve base-selective control of DNA translocation rate through aligned nanopores of the two layers. This is achieved by incorporating unpaired dangling bases called overhangs to the origami near the pore region. Molecular dynamics simulations were used to optimize the design of the origami nanopore and the overhangs. Specifically, we considered the influence of the number and spatial distribution of overhangs on translocation times. The simulations revealed that specific interactions between the overhangs and the translocating single stranded DNA resulted in base-specific residence times.
\end{abstract}

Reliable, cost effective DNA sequencing is an important and challenging goal with significant promise in the field of healthcare ${ }^{1,2}$ due to the strong correlation between the sequence and sequence modifications on disease development and progression. ${ }^{3}$ The human genome project had been carried out using Sanger method, ${ }^{4}$ which is time consuming ${ }^{5}$ and cost intensive. Further, it cannot detect post-translational modifications such as methylation, for which single molecule sequencing techniques are necessary. Nanopore based single molecule sequencing techniques have emerged as a highly promising alternative in this direction. ${ }^{6-9}$ In this technique, 
a potential difference (typically $0.1-1 \mathrm{~V}$ ) is applied across a membrane containing a nanopore (typically 2-20 nm in diameter) causing DNA to be electrophoretically driven through the pore. This process is referred as DNA translocation. The translocating DNA bases result in the modulation of ionic current passing through the pore, which can potentially be used to identify the translocating base. Nanopore sensors fall into two categories, biological and solid state nanopores. Translocation of DNA through biological nanopores such as $\alpha$-haemolysin, MspA has been extensively studied. Biological nanopores are formed by pore-forming proteins, typically a hollow core passing through a mushroom-shaped protein molecule. ${ }^{10-15}$ Biological nanopores suffer from variations in $\mathrm{pH}$, ionic strength and lack of long-term stability. ${ }^{16}$ Solid state nanopores are not affected by the $\mathrm{pH}$ or ionic strength of sample solutions and offer better control over the pore diameter. ${ }^{17-19}$ However, solid-state nanopores have higher noise levels and provide lesser control over translocation of DNA through the pore. ${ }^{20,21}$ Mono-layer graphene nanopores have been studied extensively for DNA sequencing applications ${ }^{22-25}$ due to the close match between single layer graphene thickness and the distance between two adjacent bases in a DNA strand. The matched spatial dimensions lead to better resolution of ionic current modulations due to the different bases. Extensive molecular dynamics (MD) studies have been done to understand the interactions between the translocating DNA bases and the graphene pore. ${ }^{26-29}$ Functionalization of the graphene nanopore, for instance to control the surface charge, has been explored to slow down $\mathrm{DNA}^{30-32}$ translocation, which can significantly improve the signal-to-noise ratio and thereby the base-specificity of the measured signal. ${ }^{33}$

DNA itself can be used for functionalization of solid state nanopores by virtue of base-pair interactions with the translocating strand. DNA origami offers the possibility to fabricate precise nanoscale structures which can be easily integrated with solid-state nanopores for macromolecule ${ }^{34}$ sensing as well as to control translocation process. ${ }^{35,36}$ Previously, pores made in DNA origami sheets have been combined with glass nanocapillaries for DNA trapping. ${ }^{37,38}$ 
MD studies of the dynamics of DNA origami sheet fluctuations have been done ${ }^{39,40}$. Recently, a graphene-DNA origami hybrid nanopore, consisting of sheets of DNA origami on graphene around the pore was explored by Farimani et $\mathrm{al}^{41}$. for controlling molecular transport through the nanopore system. They removed bases from the origami sheet near the nanopore to enable a "bait-prey" mechanism 34 for selective control of DNA translocation. While their work demonstrates the feasibility of such an approach, questions such as effect of number and spatial distribution of the "baits" in selective translocation control have not been addressed so far. In this study, we consider this problem by proposing a different hybrid origami-graphene nanopore system where the geometry of the nanopore and functionality of the baits are decoupled. In other words, the origami contains a nanopore region around which unpaired dangling bases, referred to as overhangs, serve as baits. The number, length and spatial distribution of the overhangs around the pore control the extent of interaction between the translocating DNA and the hybrid nanopore. The conductivity of the hybrid pores, the residence times of translocating DNA containing different base types and the mechanism of translocation were investigated in detail.

\section{Methods:}

\section{Design of the hybrid nanopore system:}

The DNA origami structures were designed using caDNAno ${ }^{42}$ software. The sheet dimensions were $138.6 \mathrm{~nm} \times 114.1 \mathrm{~nm} \times 2 \mathrm{~nm}$ with a central pore of size $3.4 \mathrm{~nm} \times 4 \mathrm{~nm}$ (Figure 1a). The graphene nanopore underneath this layer had a diameter of $2.1 \mathrm{~nm}$. The relatively larger size of the DNA origami nanopore was to allow for flexibility in the number of overhangs. The PDB (Protein Data Bank) structure was generated from the caDNAno drawing using custom written code written in Nucleic Acid Builder (NAB) ${ }^{43}$ The NAB program generates the bases first and then adds the phosphodiester bonds to get the final intended structure shown in figure 1a. Simulation was restricted to the pore region of the origami structure for ease of computation as 
well as because our study primarily focused on the interaction of the overhangs near the pore region with the translocating DNA. Figure $1 \mathrm{~b}$ shows the simulated origami sheet whose dimensions are $9.1 \mathrm{~nm} \times 8 \mathrm{~nm} \times 2 \mathrm{~nm}$. Unpaired overhangs were added to the origami staple strands near the pore region. A graphene sheet of $10 \mathrm{~nm} \times 8.5 \mathrm{~nm}$ size was generated using nanotube builder plugin of Visual Molecular Dynamics (VMD). ${ }^{44}$ A $2.1 \mathrm{~nm}$ pore was created in the graphene sheet and the DNA origami sheet was placed on top of it with both pores aligned as shown in figure 1c. In all the simulations, initially three bases of the translocating 18 mer ssDNA is inserted into the pore (figure 1d) to reduce the time involved in searching for the pore entry by the DNA strand.

Different bait configurations were evaluated by varying the length and spatial distribution of the overhangs as shown in Fig. 2a. Specifically, we included models with no bait, bait with 2 unpaired bases (henceforth referred as L2 overhang) and bait with 4 unpaired bases (henceforth referred as L4 overhang) to each of the 4 corners (Figure 2). In this study we only considered bait configurations occupying all the corners of the origami pore. In other words, 4 L2 or 4 L4 overhangs attached to each corner consisting of 8 and 16 (L2: 2 × $4=8$; L4: 4 × $4=16$ ) total unpaired bases respectively. Apart from this, a design with 10 unpaired bases arranged as a strip on either side of the origami pore (henceforth referred as LS overhang) was also considered (figure 2 c) which had 20 (LS: 2 × $10=20$ ) total unpaired bases. To understand the interactions of overhangs with the translocating DNA, we studied the translocation of 18 mer single stranded $\operatorname{poly}(\mathrm{C})_{18}$ and poly $(\mathrm{T})_{18}$ through the pore with either poly A or Poly G overhangs in the pore region. For the translocation of poly $(\mathrm{C})_{18}$ strand, 8 designs were made, namely, system with no origami sheet, origami sheet with no overhangs, L2, L4 and LS overhangs with unpaired G and A bases. The same systems were used to study the translocation of the poly $(\mathrm{T})_{18}$ strand. Table 1 provides the complete list of all configurations studied for the translocation studies. 


\section{Simulation Methodology:}

The initial structure of the origami pore with the graphene sheet and the translocating strand were loaded into xleap module of $\mathrm{AMBER}^{45}$ and solvated in a box of TIP3P water. This resulted in simulation boxes with dimensions as shown in Table 1. AMBERff $99^{46,47}$ force fields with parmbsc $0^{48,49}$ corrections (OL15) were used for describing DNA as they have been reported to reproduce the conformations of large DNA structures..$^{50}$ Joung-Chetam ion parameters ${ }^{51}$ were used for describing the interaction of the ions in the system. Particle mesh Ewald (PME) ${ }^{52}$ was used to calculate long range electrostatic interactions and a cut off of $9 \AA$ was used for shortrange interactions. SHAKE ${ }^{53}$ algorithm was used to restrain all the bonds involving hydrogen and allowed us to use an integration time step of $2 \mathrm{fs}$. The origami and the graphene sheets were initially harmonically restrained to $500 \mathrm{kcal} / \mathrm{mol} . \AA^{-2}$. To remove the bad contacts, the system was subjected to energy minimization which involved 3000 steps of steepest descent and 1000 steps of conjugate gradient while slowly reducing the harmonic restrain to zero. After the energy minimization, $40 \mathrm{ps}$ of MD simulation was performed with integration time step of $1 \mathrm{fs}$. During $\mathrm{MD}$, the system was gradually heated from $0 \mathrm{~K}$ to $300 \mathrm{~K}$ with the origami and the graphene harmonically restrained to their starting configuration using a force constant of with 20 kcal/mol.Å-2. Subsequently, equilibration of the system under constant pressure - constant temperature conditions (NPT) was performed which consisted of the following steps a) equilibration with a harmonic restrain of $1 \mathrm{kcal} / \mathrm{mol}^{-\AA^{-2}}$ for $1 \mathrm{~ns}$; b) equilibration with a harmonic restrain of $0.5 \mathrm{kcal} / \mathrm{mol} . \AA^{-2}$ for $0.5 \mathrm{~ns}$; c) equilibration with no restrain for $25 \mathrm{ps}$. Berendsen weak coupling method ${ }^{54}$ was used for both the temperature and pressure regulation with 0.5 ps time constant for heat bath coupling and 0.5 ps pressure relaxation time. For translocation simulations, $5 \mathrm{kcal} / \mathrm{mol} . \AA^{-2}$ harmonic restrain was applied to the outer corners of the origami sheet and also to the carbon atoms inside a ring of $2 \mathrm{~nm}$ radius around the pore. The system was solvated in $1 \mathrm{M}$ $\mathrm{NaCl}$ solution. The rest of the carbon atoms of the graphene sheet were fixed. 
Constant electric field was applied along $\mathrm{Z}$ direction (figure $2 \mathrm{~g}$ ) and was calculated from the voltage difference across the membrane as $\mathrm{V}=-\mathrm{E} . \mathrm{l}_{\text {trans }}\left(\mathrm{l}_{\text {trans }}\right.$ is the length of the system box along the translocation axis) and translocation simulations were performed using NAMD ${ }^{55}$ with AMBER force field parameters. The ionic current was computed by $I(t)=$ $\frac{\sum_{i=1}^{N} q_{i}\left[z_{i}(t+\Delta t)-z_{i}(t)\right]}{\Delta t * L_{z}}$, where $\mathrm{N}$ represents the total number of charge carriers contributing to the ionic current, $\Delta \mathrm{t}$ is the sampling frequency $(10 \mathrm{ps}), \mathrm{z}_{\mathrm{i}}(\mathrm{t}+\Delta \mathrm{t})$ and $\mathrm{z}_{\mathrm{i}}(\mathrm{t})$ represent the $\mathrm{z}$ coordinate of the $\mathrm{i}^{\text {th }}$ ion within the $\Delta \mathrm{t}$ and $\mathrm{q}_{\mathrm{i}}$ represents the charge of the ion. The graphical visualization presented in this study and analysis of the data was done using custom scripts written in VMD and CPPTRAJ software module of AMBER14. ${ }^{56}$

\section{Results and Discussions:}

\section{Pore characteristics:}

The conductivity of hybrid nanopores with origamis containing different bait configurations were computed. To understand the effect of the origami and baits on the nanopore conductivity, we compute the ionic current for different applied voltages. I-V characteristics for different origami designs are shown in figure 3(a). The simulations were run for $40 \mathrm{~ns}$ and the ionic current value was block averaged over the entire simulation time. It was observed that though the ions pass through the porous DNA origami sheet, they are hindered by the graphene layer. The translocating strands were not included in the simulation for computing the pore conductivity. The conductance was extracted from a linear fit of the I-V characteristics curve (figure $3 b$ ). The conductance values extracted from the simulations were $2.99 \mathrm{nS}$ (bare graphene nanopore), 2.81 $\mathrm{nS}$ (origami without baits, refer Table 1 for nomenclature of the origami types), $2.51 \mathrm{nS}$ (G2 origami, L2 with unpaired G bases), $2.13 \mathrm{nS}$ (G4 origami, L4 with unaired G bases) and $2.29 \mathrm{nS}$ (GS origami, LS with unaired G bases). The conductance of the bare graphene nanopore and that 
of the system with the origami on graphene sheet without the baits are qualitatively very similar. However, the presence of unpaired bases from the baits impedes the ionic current. Consequently, the G4 exhibits a lower conductance compared to G2, which in turn had a conductance lower than an origami with no baits. In the case of GS, despite the higher number of unpaired bases, the spatial organization of the unpaired bases makes them less flexible than that of G2 or G4. The mean variance of center of masses $\left(\sigma^{2} \mathrm{COM}\right)$ of the unpaired bases in G4 and GS was computed and was found to be $16.91 \AA^{2}$ and $9.69 \AA^{2}$ respectively for a potential difference of $2 \mathrm{~V}$ (see section S7 of supporting information). This quantitatively establishes the reduced degrees of freedom of the baits in GS despite having more unpaired bases. As a result, the GS conductance was slightly higher than the G4 value but smaller than G2. To understand the effect of the applied bias in the translocation process, we have also calculated the instantaneous electrostatic potential map (averaged over the entire simulation time) under an applied bias of $2 \mathrm{~V}$ and have shown in figure $3 \mathrm{c}$ and $3 \mathrm{~d}$. The electrostatic potential near the pore region is slightly modified because of the presence of DNA origami. For the calculation of the potential the point charges were approximated by Gaussian spheres 56 with an inverse width of $\beta=0.25 \AA$. The change in the potential profile is more for the case with a strip of overhangs than with no overhangs. We also average the potential along $\mathrm{x}$ and $\mathrm{y}$ direction and plot the total electrostatic potential along the direction of the applied bias which is along the z-direction. We find a sharp drop in the potential across the graphene nanopore and almost a constant potential across the DNA origami region. Note that similar behavior was seen the study by Farimani et. al. ${ }^{41}$ It is seen that most of the potential drop is near the graphene nanopore (figure 3e) and the type of origami did not significantly affect the potential distribution. A magnified view of the potential distribution near the nanopore is shown in figure $3 \mathrm{f}$. 


\section{SSDNA Translocation studies}

To understand the translocation of ssDNA (single stranded DNA) through the hybrid nanopore, a bias of $2 \mathrm{~V}$ was applied along the $\mathrm{z}$ axis. Eight independent trials for each configuration were run. The base is defined to have translocated when its distance along the axis of the applied electric field exceeds $3.4 \AA$ from the graphene base. Initially, 3 bases are inside the pore and so the translocation times that are calculated are effectively for 15 bases. We illustrate the role of the origami and the baits by taking the example of translocation of a ssDNA sequence poly $(\mathrm{C})_{18}$. Poly $(\mathrm{C})_{18}$ translocates across a bare graphene nanopore in a timescale of 2-4 ns, which increases to 7-9 ns on addition of the origami sheet without baits on top of graphene. This is expected due to the increased molecular interactions between the bases in the origami sheet and the translocating strand.

Addition of baits to the origami, resulted in further increase in translocation times due to basespecific interactions between the translocating $\operatorname{poly}(\mathrm{C})_{18}$ strand and the unpaired $\mathrm{G}$ bases in the bait region. The average translocation times for poly $(\mathrm{C})_{18}$ in $\mathrm{G} 2, \mathrm{G} 4$, GS were $12.09 \mathrm{~ns}, 55.43 \mathrm{~ns}$, 108.21 ns respectively in line with the increased number of unpaired bases in the bait to interact with the translocating ssDNA. The translocation times for the different configurations studied is provided in Table 2. As expected, changing the unpaired bases from $G$ to $A$ reduced the translocation time of the poly $(\mathrm{C})_{18}$ strand due to lack of base complementarity between the translocating strand and the bait. The average translocation times for A2, A4 and AS were 10.35 ns, $28.61 \mathrm{~ns}$ and $15.67 \mathrm{~ns}$ respectively. These results clearly show that incorporating an appropriate bait had a remarkable effect on the translocation time which exhibited an increasing trend with the increase in number of unpaired complementary bases in the bait region of the origami. 
To further confirm that the increase in translocation time is due to complementary base-pair interactions between the translocating strand and the bait region, we investigated the number of hydrogen bonds formed during the translocation. It was seen that the number of hydrogen bonds correlated with the residence time of the bases during translocation. In other words, an increased number of hydrogen bonds was indicative of slower translocation. This was true only when the translocating strand was complementary to the bait region as seen in table 2. Changing the bait region to unpaired A's, not complementary to the translocating poly $(C)_{18}$, resulted in a $7 x$ times decrease in the rate of hydrogen bond formation from $\sim 42$ bonds/ns to $\sim 6$ bonds/ns for the LS overhangs (More information in S3). The rate of hydrogen bond formation is the total number of hydrogen bonds formed during the complete translocation process divided by the translocation time. The statistics of hydrogen bond formation between specific and non-specific bait-target interaction is shown in Fig. 7a and 7b. Further evidence of specific base-pair interactions is obtained when the dynamics of motion of the translocating strand is analyzed for the LS system. Figure $5 \mathrm{c}$ shows that the center of mass of translocating poly $(C)_{18}$ is very close to that of one of the arms of the GS overhang, presumably due to specific C-G interaction. On the other hand, no such bias is observed when the overhang is composed of non-specific A bases. (Mechanism shown in S4)

Translocation of poly $(\mathrm{T})_{18}$ was also studied in exactly the same manner as described above for poly $(C){ }_{18}$. The results are summarized in table S6 (Supporting information) where it can be seen that the translocation behavior shows exactly similar trends as those of $\operatorname{poly}(\mathrm{C}){ }_{18}$. All these observations taken together provide confidence on our hypothesis that specific base-pair interactions strongly determine the translocation dynamics in our system. 


\section{Discussion and Open Questions:}

Although the results shown in the previous section illustrates the role of specific base interactions between the translocating strand and the unpaired bases of the overhangs, here we discuss some other factors which also affect the translocation behavior. In particular, we discuss the role of substrate interactions with the translocating strand and conformational flexibility of the baits in the bait-prey system on the translocation dynamics.

Some of the poly $(\mathrm{C})_{18}$ translocation events were found to be unusually long, particularly with G4 and GS overhangs. These have been indicated in red font in supplementary table S2. We found that such large residence times were caused due to the interaction of the translocating strand with the graphene substrate. An example of such an event is shown in the image sequence S1 (Supporting Information S1). Figure S1(f) shows the translocating strand sticking to the graphene substrate on the trans side. Such events highlight the crucial role of DNA substrate interactions on the observed translocation behavior in our system. For instance, it is possible that slowing down of the translocating strand due to stiction on graphene may enhance the rate of bait-prey interactions between the translocating strand and the origami. Vice-versa, enhanced bait-prey interactions may also increase interactions between the translocating strand and the substrate for the same reason. We are currently looking at a origami-nanopore system which can separate out bait-prey interactions from the substrate interactions. Such studies will elucidate the role of substrate in translocation behavior in our system.

As pointed earlier, the conductance of GS overhangs turns out to be larger than that of G4, in spite of larger number of unpaired bases in GS. This implies that the number of bases alone is not sufficient to understand conductance as well as translocation dynamics through the nanopore. In addition to the number of unpaired bases, flexibility of motion of the unpaired bases also appears to be an important factor. Analysis of the motion of unpaired bases revealed that LS is 
less flexible than L4 (S7). This analysis is also supported by the observation that the translocation times for the non-complementary strand was significantly higher for the L4 strand compared to the LS strand. Translocation of the non-complementary strand should not be significantly affected by the number of unpaired bases due to the lack of specific base-pair interactions. Therefore, any difference in the translocation of non-complementary strand should come from stochastic, non-specific base-pair interactions. Increase flexibility of the unpaired bases in the overhangs enhance the chance of such stochastic interactions which we believe leads to increased translocation times of the non-complementary strand translocating through L4 compared to LS. Further, in the case of a complementary strand translocation, the increase in translocation time for LS is nearly $2 \mathrm{x}$ that of L4 while the number of unpaired bases is only $25 \%$ larger. This observation is also strongly suggestive of the role of conformational flexibility of unpaired bases employed in a bait-prey system such as ours.

\section{Conclusions:}

In summary, we have studied the translocation of ssDNA through a hybrid graphene origami and demonstrated that in the design of origami pore results in tailoring the selectivity of the pore. Different origami pore designs on graphene were evaluated for their effect on translocation of complementary and non-complementary strands. It was found that complementary interactions between translocating strand and unpaired bases (baits) in the origami resulted in significantly longer translocation time correlating with increased rate of hydrogen bond formation. While this was expected, we also found evidence for the role of substrate-DNA interactions and conformational flexibility of the baits in determining translocation behavior. Our studies provide insight into optimal design of hybrid DNA origami nanopore structures for sensing and sequencing. 


\section{Acknowledgement}

We thank DBT, India for financial support. We acknowledge Supercomputer Education and Research Centre, (SERC) IISc Bangalore for providing access to the high-performance supercomputer SahasraT. R.K and S.P. thank MHRD, GoI for research fellowship. We also acknowledge the use of facilities at Centre for Nano Science and Engineering, Indian Institute of

Science, Bangalore, India. We also thank Prof. Anjana Rao of La Jolla Institute for Allergy and Immunology for useful discussions.

\section{References:}

1. Shendure, J. et al. DNA sequencing at 40: past, present and future. Nature 550, 345-353 (2017).

2. Goodwin, S., McPherson, J. D. \& Richard McCombie, W. Coming of age: ten years of next-generation sequencing technologies. Nat. Publ. Gr. 17, (2016).

3. Levinson, D. F. The Genetics of Depression: A Review. Biol. Psychiatry 60, 84-92 (2006).

4. Sanger, F., Nicklen, S. \& Coulson, A. R. DNA sequencing with chain-terminating inhibitors. Proc. Natl. Acad. Sci. U. S. A. 74, 5463-7 (1977).

5. Tyler F. Beck, James C. Mullikin, Leslie G. Biesecker. Systematic Evaluation of Sanger Validation of NextGen Sequencing Variants. 62, 647-654 (2016).

6. Deamer, D. W. \& Branton, D. Characterization of Nucleic Acids by Nanopore Analysis The Nanopore Concept. doi:10.1021/ar000138m

7. Venkatesan, B. M. \& Bashir, R. Nanopore sensors for nucleic acid analysis. Nat. Nanotechnol. 6, 615-24 (2011).

8. Keyser, U. F. \& Keyser, U. F. Controlling molecular transport through nanopores Controlling molecular transport through nanopores. 1369-1378 (2011). doi:10.1098/rsif.2011.0222

9. Lansac, Y., Maiti, P. K. \& Glaser, M. A. Coarse-grained simulation of polymer translocation through an artificial nanopore. Polymer (Guildf). 45, 3099-3110 (2004).

10. Kasianowicz, J. J., Brandin, E., Branton, D. \& Deamer, D. W. Characterization of individual polynucleotide molecules using a membrane channel. Proc. Natl. Acad. Sci. U. S. A. 93, 13770-3 (1996).

11. Akeson, M., Branton, D., Kasianowicz, J. J., Brandin, E. \& Deamer, D. W. Microsecond Time-Scale Discrimination Among Polycytidylic Acid, Polyadenylic Acid, and 
Polyuridylic Acid as Homopolymers or as Segments Within Single RNA Molecules. Biophys. J. 77, 3227-3233 (1999).

12. Cockroft, S. L., Chu, J., Amorin, M., Ghadiri, M. R. \& Ghadiri, M. R. A single-molecule nanopore device detects DNA polymerase activity with single-nucleotide resolution. $J$. Am. Chem. Soc. 130, 818-20 (2008).

13. Derrington, I. M. et al. Nanopore DNA sequencing with MspA. Proc. Natl. Acad. Sci. U. S. A. 107, 16060-5 (2010).

14. Manrao, E. A. et al. Reading DNA at single-nucleotide resolution with a mutant MspA nanopore and phi29 DNA polymerase. Nat. Biotechnol. 30, 349-353 (2012).

15. Kumar, H., Lansac, Y., Glaser, M. A. \& Maiti, P. K. Biopolymers in nanopores: challenges and opportunities. Soft Matter 7, 5898 (2011).

16. Haque, F., Li, J., Wu, H.-C., Liang, X.-J. \& Guo, P. Solid-State and Biological Nanopore for Real-Time Sensing of Single Chemical and Sequencing of DNA. Nano Today 8, 5674 (2013).

17. Storm, A. J., Chen, J. H., Ling, X. S., Zandbergen, H. W. \& Dekker, C. Fabrication of solid-state nanopores with single-nanometre precision. Nat. Mater. 2, 537-540 (2003).

18. Venkatesan, B. M. et al. Highly Sensitive, Mechanically Stable Nanopore Sensors for DNA Analysis. Adv. Mater. 21, 2771 (2009).

19. Kim, M. J., Wanunu, M., Bell, D. C. \& Meller, A. Rapid Fabrication of Uniformly Sized Nanopores and Nanopore Arrays for Parallel DNA Analysis. Adv. Mater. 18, 3149-3153 (2006).

20. Branton, D. et al. The potential and challenges of nanopore sequencing. Nat. Biotechnol. 26, 1146-1153 (2008).

21. Iqbal, S. M., Akin, D. \& Bashir, R. Solid-state nanopore channels with DNA selectivity. Nat. Nanotechnol. 2, 243-8 (2007).

22. Geim, A. K. Graphene: Status and Prospects. Science (80-. ). 324, 1530-1534 (2009).

23. Fischbein, M. D. \& Drndić, M. Electron beam nanosculpting of suspended graphene sheets. Appl. Phys. Lett. 93, 113107 (2008).

24. Merchant, C. A. et al. DNA Translocation through Graphene Nanopores. Nano Lett. 10, 2915-2921 (2010).

25. Schneider, G. F. \& Dekker, C. DNA sequencing with nanopores. Nat. Biotechnol. 30, 326-328 (2012).

26. Wells, D. B., Belkin, M., Comer, J. \& Aksimentiev, A. Assessing graphene nanopores for sequencing DNA. Nano Lett. 12, 4117-4123 (2012).

27. Qiu, H., Sarathy, A., Leburton, J. P. \& Schulten, K. Intrinsic Stepwise Translocation of Stretched ssDNA in Graphene Nanopores. Nano Lett. 15, 8322-8330 (2015).

28. Sathe, C., Zou, X., Leburton, J. \& Schulten, K. Computational Investigation of DNA 
Detection Using Graphene Nanopores. 8842-8851 (2011).

29. Liang, L. Computational studies of DNA sequencing with graphene nanopores. (2014).

30. Banerjee, S. et al. Slowing DNA transport using graphene-DNA interactions. Adv. Funct. Mater. 25, 936-946 (2015).

31. Luan, B., Stolovitzky, G. \& Martyna, G. Slowing and controlling the translocation of DNA in a solid-state nanopore. Nanoscale 4, 1068-77 (2012).

32. Sint, K., Wang, B. \& Král, P. Selective Ion Passage through Functionalized Graphene Nanopores. J. Am. Chem. Soc. 130, 16448-16449 (2008).

33. Yu, Y.-S., Lu, X., Ding, H.-M. \& Ma, Y.-Q. Computational investigation on DNA sequencing using functionalized graphene nanopores. Phys. Chem. Chem. Phys doi:10.1039/c7cp07462j

34. Wei, R., Martin, T. G., Rant, U. \& Dietz, H. DNA Origami Gatekeepers for Solid-State Nanopores. Angew. Chemie Int. Ed. 51, 4864-4867 (2012).

35. Winfree, E., Liu, F., Wenzler, L. A. \& Seeman, N. C. Design and self-assembly of twodimensional DNA crystals. Nature 394, (1998).

36. Rothemund, P. W. K. Folding DNA to create nanoscale shapes and patterns. Nature 440, 297-302 (2006).

37. Hernandez-Ainsa, S. et al. DNA origami nanopores for controlling DNA translocation. ACS Nano 7, 6024-6030 (2013).

38. Keyser, U. F. Enhancing nanopore sensing with DNA nanotechnology. Nat. Nanotechnol. 11, 106-108 (2016).

39. Yoo, J. \& Aksimentiev, A. In situ structure and dynamics of DNA origami determined through molecular dynamics simulations. Proc. Natl. Acad. Sci. U. S. A. 110, 20099-104 (2013).

40. Li, C.-Y. et al. Ionic Conductivity, Structural Deformation, and Programmable Anisotropy of DNA Origami in Electric Field. ACS Nano 9, 1420-1433 (2015).

41. Barati Farimani, A., Dibaeinia, P. \& Aluru, N. R. DNA Origami-Graphene Hybrid Nanopore for DNA Detection. ACS Appl. Mater. Interfaces acsami.6b11001 (2016). doi:10.1021/acsami.6b11001

42. Douglas, S. M. et al. Rapid prototyping of 3D DNA-origami shapes with caDNAno. Nucleic Acids Res. 37, 5001-5006 (2009).

43. Macke, T. J. \& Case, D. A. Modeling Unusual Nucleic Acid Structures. in 379-393 (1997). doi:10.1021/bk-1998-0682.ch024

44. Humphrey, W., Dalke, A. \& Schulten, K. VMD: visual molecular dynamics. J. Mol. Graph. 14, 33-8, 27-8 (1996).

45. Case, D. A. et al. The Amber biomolecular simulation programs. J. Comput. Chem. 26, 1668-1688 (2005). 
46. Cornell, W. D. et al. A Second Generation Force Field for the Simulation of Proteins, Nucleic Acids, and Organic Molecules. J. Am. Chem. SOC 117, 5179-5197 (1995).

47. Cheatham, T. E., Cieplak, P. \& Kollman, P. A. A Modified Version of the Cornell et al. Force Field with Improved Sugar Pucker Phases and Helical Repeat. J. Biomol. Struct. Dyn. 16, 845-862 (1999).

48. Pérez, A. et al. Refinement of the AMBER Force Field for Nucleic Acids: Improving the Description of $\alpha / \gamma$ Conformers. Biophys. J. 92, 3817-3829 (2007).

49. Dans, P. D. et al. How accurate are accurate force-fields for B-DNA? Nucleic Acids Res. 45, gkw1355 (2017).

50. Maiti, P. K., Pascal, T. A., Vaidehi, N. \& Goddard, W. A. The stability of Seeman JX DNA topoisomers of paranemic crossover (PX) molecules as a function of crossover number. Nucleic Acids Res. 32, 6047-6056 (2004).

51. Joung, I. S. \& Cheatham, T. E. Determination of Alkali and Halide Monovalent Ion Parameters for Use in Explicitly Solvated Biomolecular Simulations. J. Phys. Chem. B 112, 9020-9041 (2008).

52. Darden, T., York, D. \& Pedersen, L. Particle mesh Ewald: An $N \cdot \log (N)$ method for Ewald sums in large systems. J. Chem. Phys. 98, 10089-10092 (1993).

53. Ryckaert, J.-P., Ciccotti, G. \& Berendsen, H. J. . Numerical integration of the cartesian equations of motion of a system with constraints: molecular dynamics of n-alkanes. $J$. Comput. Phys. 23, 327-341 (1977).

54. Berendsen, H. J. C., Postma, J. P. M., van Gunsteren, W. F., DiNola, A. \& Haak, J. R. Molecular dynamics with coupling to an external bath. J. Chem. Phys. 81, 3684-3690 (1984).

55. Phillips, J. C. et al. Scalable molecular dynamics with NAMD. J. Comput. Chem. 26, 1781-1802 (2005).

56. Roe, D. R. \& Cheatham, T. E. PTRAJ and CPPTRAJ: Software for Processing and Analysis of Molecular Dynamics Trajectory Data. J. Chem. Theory Comput. 9, 3084-3095 (2013). 